\title{
Correction to: geometry assessment of ultra-short pulsed laser drilled micro-holes
}

\author{
Matthias Putzer ${ }^{1,2} \cdot$ Norbert Ackerl $^{2} \cdot$ Konrad Wegener ${ }^{2}$ \\ Published online: 19 January 2021 \\ (C) Springer-Verlag London Ltd., part of Springer Nature 2021
}

\section{Correction to: The International Journal of Advanced Manufacturing Technology. https://doi.org/10.1007/s00170-020-06199-5}

The original article contained a mistake.

Section 1 Introduction

- The unit of power density $\left(\mathrm{W} \mathrm{cm} \mathrm{s}^{-2}\right)$ is incorrect. Correctly, it must be $\mathrm{W} \mathrm{cm}^{-2}$.

Section 2.1 Material and machine

- The dash in 515-nm is not correct. Correctly, it must be $515 \mathrm{~nm}$.

Section 2.2 Circular path laser drilling

- The $b$ in the formula symbol of hatching distance $l b$. is not subscripted. Correctly, it must be $l_{b}$.

- The path of the laser beam is termed by $\mathrm{d}_{\text {hatch }}$. Correctly, it must be $d_{\text {path }}$ as in Fig. 1 .

- The total diameter of the surface irradiated with the laser is termed by $\mathrm{d}_{\text {hole }}$. Correctly, it must be $\mathrm{d}$ as in Fig. 1 .

- The eq. (1) is incorrect. Correctly it must be $d_{p a t h}=d-d_{f o c}$

Caption in Fig. 1.

The FOV in the formula symbol of the length of the field of view $1 F O V$ is not subscripted. Correctly, it must be $1_{F O V}$.

The online version of the original article can be found at https://doi.org/ $10.1007 / \mathrm{s} 00170-020-06199-5$

\section{Matthias Putzer}

putzer@iwf.mavt.ethz.ch

inspire AG, Technoparkstrasse 1, Zurich, Switzerland

2 Department of Mechanical Engineering, IWF, ETH Zurich, Leonhardstrasse 21, Zurich, Switzerland
Section 3.4 Through-hole of $300 \mu \mathrm{m}$ depth.

The dash in $300-\mu \mathrm{m}$ is not correct. Correctly, it must be $300 \mu \mathrm{m}$.

Revised version of the bibliography in, as the current version is not consistent in the referencing style of the individual sources:

1 Ackerl N, Boerner P, Wegener K (2019) Toward application of hierarchical structures by ultrashort pulsed laser ablation. J Laser Appl 31:022501. https://doi.org/10.2351/1.5096079

2 Shugaev M,Wu C, Armbruster O, Naghilou A, Brouwer N, Ivanov D, Derrien T-Y, Bulgakova N, Kautek W, Rethfeld B, Zhigilei L (2016) Fundamentals of ultrafast laser-material interaction. MRS Bull 41(12):960-968. https://doi.org/10.1557/ mrs.2016.274

3 Ackerl N, Wegener K (2019) Ablation characteristics of alumina and zirconia ceramics on ultra-short pulsed laser machining. J Laser Micro Nanoen 14(2):168-172. https://doi. org/10.2961/jlmn.2019.02.0009

4 Carvalho A, Grenho L, Fernandes M, Daskalova A, Trifonov A, Buchvarov I, Monteiro F (2020) Femtosecond laser microstructuring of alumina toughened zirconia for surface functionalization of dental implants. Ceram Int 46(2):1383-1389. https://doi.org/10.1016/j.ceramint.2019. 09.101

5 Ackerl N, Warhanek M, Gysel J, Wegener K (2019) Ultra short pulsed laser machining of dental ceramic implants. J Eur Ceram Soc 39(4):1635-1641. https://doi.org/10.1016/j. jeurceramsoc.2018.11.007

6 Itoh K (2014) Ultrafast laser processing of glass. J Laser Micro Nanoen 9(3):187-191. https://doi.org/10.2961/jlmn. 2014.03.0001

7 Murakami R, Nakagawa H, Matsuo S (2017) Water-assisted laser drilling for miniature internal thread in glass and evaluation of its strength. J Laser Micro Nanoen 12(3):203-206. https://doi.org/10.2961/jlmn.2017.03.0005 
8 Walter C, Komischke T, Kuster F, Wegener K (2014) Laser structured grinding tools - generation of prototype patterns and performance evaluation. J Mater Process Tech 214(4):951-961. https://doi.org/10.1016/j.jmatprotec.2013.11.015

9 Warhanek M,Walter C, Hirschi M, Boos J, Bucourt JF,Wegener K (2016) Comparative analysis of tangentially laser-processed fluted polycrystalline diamond drilling tools. J Manuf Process 23:157- 164. https://doi.org/10.1016/j. jmapro.2016.06.023

10 Chong TC, Hong MH, Shi LP (2010) Laser precision engineering: from microfabrication to nanoprocessing. Laser Photonics Rev 4(1):123-143. https://doi.org/10.1002/lpor. 200810057

11 Warhanek M, Mayr J, Dörig C, Wegener K (2017) Accurate micro-tool manufacturing by iterative pulsed-laser ablation. Lasers Manuf Mater Process 4:193-204. https:// doi.org/10.1007/s40516-017-0046-y

12 Giedl R, Helml H-J, Wagner FX, Wild MJ (2003) Geometrical aspects of laser-drilled high precision holes for flow control applications. Proceedings SPIE 5063, Fourth International Symposium on Laser Precision Microfabrication. https://doi.org/10.1117/12.540609

13 Weck A, Crawford T, Wilkinson D, Haugen H, Preston J (2008) Laser drilling of high aspect ratio holes in copper with femtosecond, picosecond and nanosecond pulses. J Appl Phys A 90:537-543. https://doi.org/10.1007/s00339007-4300-6

14 Mincuzzi G, Faucon M, Kling R (2019) Novel approaches in zero taper, fast drilling of thick metallic parts by ultra-short pulse laser. Opt Lasers Eng 118:52-57. https://doi.org/10. 1016/j.optlaseng.2019.03.004

15 Gruner A, Naumann L, Schille J, Loeschner U (2019) High pulse repetition frequency micro hole drilling of silicon using ultrashort pulse laser radiation. J LaserMicro Nanoen 14(3):190-197. https://doi.org/10.2961/jlmn.2019.03.0001

16 Ancona A, Döring S, Jauregui C, Röser F, Limpert J, Nolte S, Tünnermann A (2009) Femtosecond and picosecond laser drilling of metals at high repetition rates and average powers. Opt Lett 34(21):3304-3306. https://doi.org/10.1364/OL.34.003304

17 Ashkenasi D, Kaszemeikat T, Mueller N, Dietrich R, Eichler HJ, Illing G (2011) Laser trepanning for industrial applications. Phys Procedia 12:323-331. https://doi.org/10. 1016/j.phpro.2011.03.140

18 Celia E, Darmanin T, Taffin de Givenchy E, Amigoni S, Guittard F (2013) Recent advances in designing superhydrophobic surfaces. J Colloid Interf Sci 402:1-18. https://doi.org/10.1016/j.jcis.2013.03.041

19 Zheng C, Zhao K, Shen H, Zhao X, Yao Z (2020) Crack behavior in ultrafast laser drilling of thermal barrier coated nickel superalloy. J Mater Process Tech 282:116678. https:// doi.org/10.1016/j.jmatprotec.2020.116678

20 Antar M, Chantzis D,Marimuthu S, Hayward P (2016) High speed EDM and laser drilling of aerospace alloys. Proc
Cirp 42:526-531. https://doi.org/10.1016/j.procir.2016.02. 245

21 Mc Clure S, King J, Koivula J, Moses T (2000) A new lasering technique for diamond. Gems Gemol 36(2):138-146. https://doi.org/10.5741/GEMS.36.2.138

22 Nasrollahi V, Penchev P, Batal A, Le H, Dimov S, Kim K (2020) Laser drilling with a top-hat beam of micro-scale high aspect ratio holes in silicon nitride. J Mater Process Tech 281:1166368. https://doi.org/10.1016/j.jmatprotec.2020. 116636

23 Mottay E, Liu X, Zhang H, Mazur E, Sanatinia R, Pfleging W (2016) Industrial applications of ultrafast laser processing. MRS Bull 41(12):984-992. https://doi.org/10.1557/mrs. 2016.275

24 Schneller E, Dhere NG, Kar A (2014) Overview of laser processing in solar cell fabrication. Spie Proc Ser 9180:918002. https://doi.org/10.1117/12.2062023

25 Qin Z, Xiang H, Liu J, Zeng X (2019) High-performance oil- water separation polytetrafluoroethylene membranes prepared by picosecond laser direct ablation and drilling. Mater Design 184:108200. https://doi.org/10.1016/j.matdes.2019. 108200

26 Yan S, Ren F, Li C, Jiao Y,Wang C,Wu S, Wei S, Hu Y, Li J, Xiao Y, Su Y, Wu D (2018) Unidirectional self-transport of air bubble via a Janus membrane in aqueous environment. Appl Phys Lett 113(26):261602. https://doi.org/10.1063/1. 5052566

27 Jianguo M, Lisheng D, Guanzhong Y (2000) Laser drilling with high speed for osmotic drug delivery system. Chin Pharm J 35(1): 47-49

28 Veenstra FLP, Ackerl N, Martín AJ, Pérez-Ramírez J (2020) Lasermicrostructured copper reveals selectivity patterns in the electrocatalytic reduction of $\mathrm{CO} 2$. Chem 6(7):1707-1722. https://doi.org/10.1016/j.chempr.2020.04. 001

29 Ackerl N, Gysel J, Warhanek M, Wegener K (2019) Ultrashort pulsed laser manufacturing of yttria stabilized aluminatoughened zirconia dental implants. Proc SPIE 10857:108570J, Lasers in Dentistry XXV. https://doi.org/10. $1117 / 12.2507495$

30 Haynes WM, David RL, Bruno TJ (2017) Handbook of chemistry and physics, 97th edn. In: CRC Press Taylor \& Francis

31 Liu JM (1982) Simple technique for measurements of pulsed Gaussian-beam spot sizes. Opt Lett 7(5):196-198. https://doi.org/10.1364/OL.7.000196

32 Nolte S,Momma C, Jacobs H, TuA, Chichkov BN, Wellegehausen B,Welling H (1997) Ablation of metals by ultrashort laser pulses. J Opt Soc Am B 14(10):2716-2722. https://doi.org/10.1364/JOSAB.14. 002716

33 Förster DJ, Weber R, Holder D, Graf T (2018) Estimation of the depth limit for percussion drilling with picosecond laser 
pulses. Opt Express 26(9):11546. https://doi.org/10.1364/OE. 26.011546

34 Weber R, Graf T, Freitag C, Feuer A, Kononenko T, Konov VI (2017) Processing constraints resulting from heat accumulation during pulsed and repetitive laser materials processing. Opt Express 25(4):3966-3979. https://doi.org/10. 1364/OE.25.003966
The original article has been corrected.

Publisher's note Springer Nature remains neutral with regard to jurisdictional claims in published maps and institutional affiliations. 